\title{
How to switch on and switch off semantic priming effects for natural and artifactual categories: activation processes in category memory depend on focusing specific feature dimensions
}

\author{
Christina Bermeitinger • Dirk Wentura • \\ Christian Frings
}

Published online: 5 March 2011

(C) Psychonomic Society, Inc. 2011

\begin{abstract}
Semantic priming" refers to the phenomenon that people react faster to target words preceded by semantically related rather than semantically unrelated words. We wondered whether momentary mind sets modulate semantic priming for natural versus artifactual categories. We interspersed a category priming task with a second task that required participants to react to either the perceptual or action features of simple geometric shapes. Focusing on perceptual features enhanced semantic priming effects for natural categories, whereas focusing on action features enhanced semantic priming effects for artifactual categories. In fact, significant priming effects emerged only for those categories thought to rely on the features activated by the second task. This result suggests that (a) priming effects depend on momentary mind set and (b) features can be weighted flexibly in concept representations; it is also further evidence for sensory-functional accounts of concept and category representation.
\end{abstract}

Keywords Semantic priming - Category specificity · Natural categories · Artifactual categories - Semantic knowledge $\cdot$ Semantic memory Concept representation . Mind set - Task set

\footnotetext{
C. Bermeitinger $(\bowtie)$

Department of Psychology, University of Hildesheim,

Marienburger Platz 22,

31141 Hildesheim, Germany

e-mail: bermeitinger@uni-hildesheim.de

C. Bermeitinger $\cdot$ D. Wentura $\cdot$ C. Frings

Saarland University,

Saarbrücken, Germany
}

How semantic knowledge is represented in the human brain is a prevailing theme in psychology and cognitive neuroscience. The most utilized paradigm in cognitive psychology for studying the organization and processing of semantic knowledge is the semantic priming paradigm (for reviews, see McNamara, 2005; Neely, 1991). Typically, participants judge whether or not a target word is a real word, and a prime word precedes each target. Prime and target can be semantically related (e.g., flower-rose) or semantically unrelated (e.g., bird-rose). Although primes are completely irrelevant to the participants' task and do not predict the correct target response, participants usually respond faster in semantically related than in semantically unrelated trials (i.e., the semantic priming effect). Semantic priming is often explained in terms of spreading activation or a transition from the prime representation to the target representation (e.g., Anderson, 1983; Collins \& Loftus, 1975; Masson, 1995; Rogers \& McClelland, 2004; see McNamara, 2005, for a review). Yet a tacit assumption underlying the theories is that from priming effects (or their absence) one can infer principles of inflexible and fixed organization within semantic memory (cf. McNamara, 2005). If no semantic priming effect for a specific type of relationship between a prime and a target is found, it is typically concluded that this particular relationship is not relevant for the organization of semantic memory. In this article, we challenge the assumption that a static organization within semantic memory is measured by semantic priming; instead, we argue that momentary mind sets modulate semantic priming, thereby yielding evidence for the dynamic representation of concepts. Momentary mind sets are global settings or orientations for processing information in a specific way (see, e.g., Gollwitzer \& 
Kinney, 1989; Martens \& Kiefer, 2009; Rothermund, Voss, \& Wentura, 2008; Spruyt, De Houwer, \& Hermans, 2009).

With regard to processing semantic information, Kiefer (2007; see also Kiefer \& Martens, 2010) suggested that such mind sets can modulate, in a top-down fashion, which particular aspects of semantic information are processed. For example, mind sets might consist of dominantly focusing on a broad category of features - for example, perceptual or functional features - for a longer period (e.g., a whole experiment). In fact, neuropsychological studies on category-related semantic processing have found evidence for the flexible recruitment of perceptual and action-related conceptual features in sensory and motor areas (Hoenig, Sim, Bochev, Herrnberger, \& Kiefer, 2008; Sim \& Kiefer, 2005; see also Martin \& Chao, 2001). Although these studies used an experimental task different from ours (i.e., repetition priming), they yielded evidence that concepts can be flexibly recruited, depending on mind set. ${ }^{1}$

Turning to semantic priming, only three studies have yielded tentative evidence for the modulation of semantic priming due to mind sets. The first study was run by McKoon and Ratcliff (1995). They found that semantic priming of a particular relationship (e.g., synonym or antonym) depended on the proportion of trials in the experiment sharing this relationship. For example, significant antonym priming (e.g., close-far) was found only when a high proportion of trials included an antonym relationship, not when a high proportion of trials included a synonym relationship. Indeed, McKoon and Ratcliff emphasized the potential challenge of these results for "static assumptions" of theories on semantic priming. However, as a consequence of their "mind set" manipulation, McKoon and Ratcliff's study featured an extreme relatedness proportion (i.e., the proportion of related primetarget pairs) and an extreme nonword ratio (i.e., the majority of the unrelated prime-target pairs contained a nonword target). Thus, their effects are suspected to be the result of strategic processes (see Hutchison, 2003, for a detailed critique of these issues). The second study was run by Bermeitinger, Wentura, and Frings (2008), who obtained evidence that semantic priming effects for natural and artifactual categories may be modulated by the experimental context. In a nutshell, semantic priming effects in female participants were affected by a manipulation that could tentatively be interpreted as emphasizing either perceptual or functional features (thereby possibly inducing a "per-

\footnotetext{
${ }^{1}$ We hasten to add that such mind sets should not be misunderstood as the context-dependent activation of single features of specific concepts (e.g., Barsalou, 1982; Pecher, Zeelenberg, \& Raaijmakers, 1998; Yeh \& Barsalou, 2005). For example, "piano" primes either its property "heavy" or "instrument," depending on whether it is introduced in a context of "moving" or "playing music" (see Greenspan, 1986). Mind sets are more abstract and enduring states.
}

ceptual mind set" or a "functional mind set"). The third study was recently conducted by Kiefer and Martens (2010; see also Martens \& Kiefer, 2009, for behavioral data). These authors presented masked primes before unmasked targets within a lexical decision task. With a semantic task set, a semantic priming effect was found in the electroencephalogram (EEG) measure (i.e., the N400 component). However, with a perceptual task set, no priming was observed.

We suggest that semantic priming can generally be modulated by participants' mind sets. To this end, we developed a new experimental procedure for inducing mind sets that also avoids the mentioned confound of mind-set manipulation and relatedness proportion (McKoon \& Ratcliff, 1995). We used a category priming task with natural and artifactual categories. There is consensus that natural categories are mainly represented according to their perceptual features, whereas artifactual categories are mainly represented according to their functional features (see, e.g., McRae \& Cree, 2002). Thus, we interspersed two secondary tasks (referred to as "focus tasks" in the following) in a category priming task to shift participants' attention to either perceptual or action-related features. To ensure that we were dealing with a general and abstract mind set (and not context-specific activation), we meticulously designed the focus tasks to meet the following criteria: They should be nonverbal and completely independent of the semantic priming material, and the perception and action focus tasks should be of comparable difficulty, use the same materials, and be perceptually identical. In focus-task trials, a matrix containing different symbols in some cells was presented. Participants were requested to attend either to the identity of a specific symbol (perception focus) or to the direction of a potential symbol movement (action focus). If the interspersed focus trials induced a general mind set, we would expect an interaction of category type and focus task. In particular, participants with a perceptual focus should show semantic priming for natural categories but not for artifactual categories, whereas participants with an action focus should show semantic priming for artifactual categories but not for natural categories.

\section{Method}

\section{Participants}

Forty-five paid students ( 21 female; median age 22 years, range 17-34) from Saarland University participated in the experiment. The data of 2 further participants were discarded; 1 showed an outlying error rate in both tasks (more than 35\% errors overall), and the other was a 
Fig. 1 (a) Procedure of a priming trial, with an example of an unrelated prime-target pair. (b) Cue assignment and two examples of matrices used for the focus tasks. For the perception focus task, a symbol was cued by a specific color, and participants had to identify the corresponding movable symbol. (Here, "movable" means that there is at least one empty cell located next to a cell filled with the symbol.) Thus, shape (of the symbols) was the task-relevant dimension. For the action focus task, a specific direction was cued by the color, and participants had to identify the goal cell of the only pushable symbol in the matrix, which could be moved in the cued direction by pushing it from the other side. (Here, "pushable" means that at least one empty cell is on either side of the symbol cell). Thus, the focus is on accomplishing an action (irrespective of the

shape). In the upper figures, the solution is indicated in red, and in the lower figures, the solution is indicated by upside-down text. Note that the red markings/ upside-down text, as well as the labels for the cues, the row and column numbers, and the question displayed above the first matrix, are for illustrative purposes only and did not appear in the actual experiment. Whether participants should respond to the row or the column of the goal cell was printed below each matrix a

\section{Priming task}

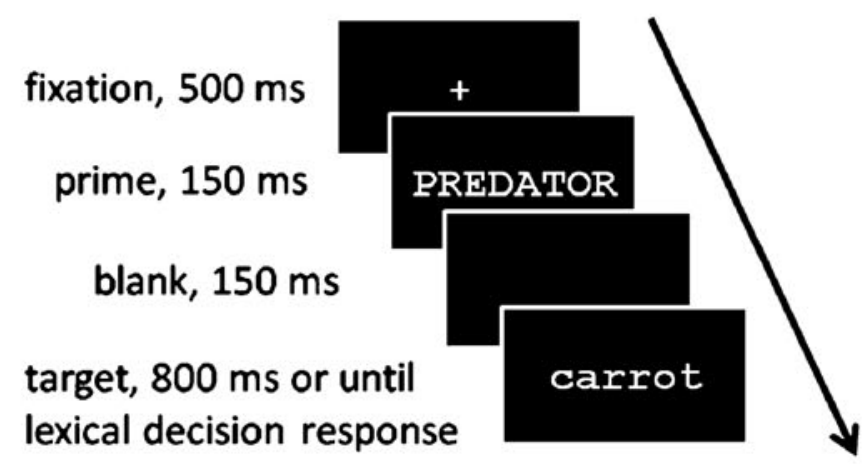

b

Focus task

Perception Action

Cue assignment

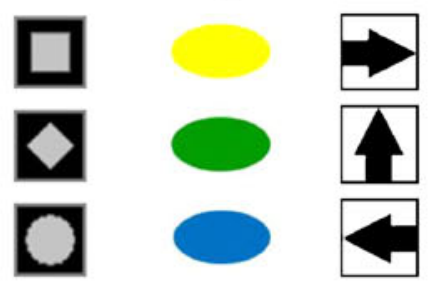

\section{Matrix task}

In which row is the symbol

(which can be moved)

containing the cued shape?

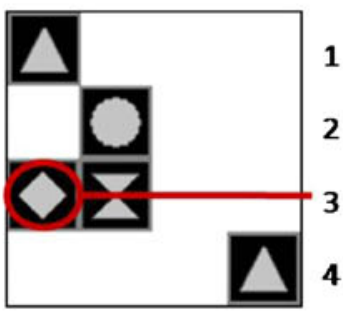

$\begin{array}{llll}1 & 2 & 3 & 4\end{array}$ ROW?
Which row is the goal row of the symbol that can be pushed towards the cued direction?
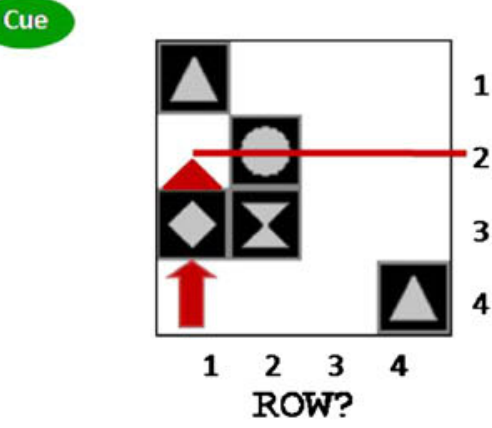

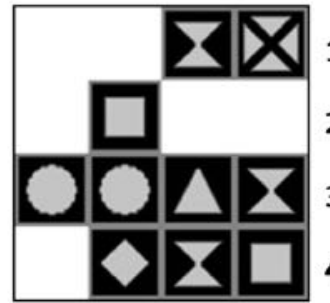

$\begin{array}{llll}1 & 2 & 3 & 4\end{array}$ COLUMN?

I : jamsue panos auo uunjos

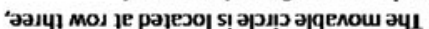
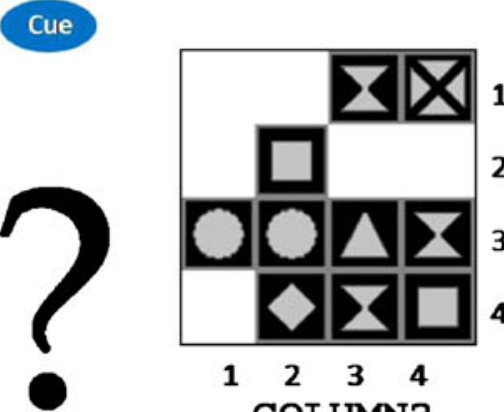

$\begin{array}{llll}1 & 2 & 3 & 4\end{array}$ COLUMN?
I Lamsue payoj auo uun|03 o] spuem-yə paysnd aq ues om 1 mod u! ajenbs aч 1 
multivariate outlier, as indicated by Mahalanobis distance (e.g., Stevens, 1984).

\section{Design}

Essentially, we used a 2 (focus task: perception vs. action) $\times 2$ (category type: natural vs. artifactual) $\times 2$ (priming condition: related vs. unrelated prime-target pair) design. Additionally, the factor Target Orthography (word vs. nonword) was also included. Apart from the focus task, the factors were varied within subjects, with the factor Category Type varied blockwise. Semantic priming effects were computed as the difference in mean reaction times (RTs) between related and unrelated trials.

\section{Materials}

The priming task was a word-nonword decision task (i.e., a lexical decision task). The materials for the decision task were the same as those reported in Bermeitinger et al. (2008). We used four natural category labels and four artifactual category labels as primes. Additionally, neutral primes (i.e., strings of seven random letters) were used in filler trials to lower the overall proportion of related trials. Six category exemplars from each category served as word targets. For the word-nonword decision task, pronounceable nonwords were created by changing one letter of each word target. The natural and artifactual primes and targets did not differ with respect to mean association frequency (i.e., dominance), mean length, mean word frequency, or category size (all $p \mathrm{~s}>.05$ ). All stimuli were presented in light gray on a black screen and were approximately $0.5 \mathrm{~cm}$ in height.

For the focus tasks, 36 four-by-four matrices were created (length $4 \mathrm{~cm}$, height $4 \mathrm{~cm}$ ). In each matrix, a varying number of cells contained symbols (for some examples, see Fig. 1b). The symbol for each trial was selected from a set of eight symbols. The construction of matrices allowed using each matrix for both tasks. An additional set of 24 matrices was used for practice.

\section{Procedure}

Participants were individually tested in sound-attenuating chambers. The experiment was run using E-Prime software (version 1.3) with a standard PC and 17-in. CRT monitors. Viewing distance was about $60 \mathrm{~cm}$, and instructions were given on screen.

The experiment proper featured two blocks, one featuring natural and one featuring artifactual category primes (counterbalanced across participants). Each block consisted of three subblocks containing 48 priming trials (16 related, 16 unrelated, and 16 neutral prime-target pairs; half of the trials included nonword targets) and 12 focus trials. Before the first block, participants worked through several focus task practice blocks. Before each block, participants performed practice trials for, first, the priming task and, second, the combined priming and focus task.

The main part of the experiment consisted of semantic priming trials, which were mixed with focus trials. In the priming trials (see Fig. 1a), a fixation cross first appeared at the center of the screen for $500 \mathrm{~ms}$. This was followed by the prime $(150 \mathrm{~ms})$ written in capital letters and a blank screen $(150 \mathrm{~ms}$; thus, the SOA was $300 \mathrm{~ms})$. The target then appeared and remained on the screen until a response was given or until $800 \mathrm{~ms}$ had elapsed. Erroneous responses and responses slower than $800 \mathrm{~ms}$ produced an error or feedback message (reminding participants to respond more quickly), respectively. The intertrial interval was 1,000 ms. Participants were requested to quickly and accurately categorize each target word with regard to its orthography.

A focus trial consisted of a colored ellipse (which served as cue) and a matrix. In cue-learning blocks before the first priming block, participants learned to assign each of three colors to either a specific shape (perception focus) or a specific direction (action focus; see Fig. 1b). In the main part of the experiment, each subblock started with a cue for $2,000 \mathrm{~ms}$, followed by a blank screen for $150 \mathrm{~ms}$. After a random number of priming trials (at least one), a blank screen appeared for $150 \mathrm{~ms}$, followed by a matrix until a response was given. Each matrix appeared together with the information about whether the row or the column of the response cell (defined by the cued shape or the cued direction) should be specified by a press on a labeled key. After an erroneous or correct response was given, the border of the matrix became red or white, respectively, before the matrix was erased. In the perception focus task, participants had to specify either the row or the column of the symbol that was assigned to the color and that was movable (e.g., the diamond on the left, cued by the green cue, can be either moved upward or downward; see Example 1 in Fig. 1b). For the perception focus task, "movable" meant that at least one white space was located next to the box filled with the cued symbol, independent of whether the white space was located to the left/right or above/below. In the action focus task, participants had to find the symbol that was pushable in the direction associated with the cue. A specific box was defined as being pushable if white spaces were located on both sides of it. In this sense, within each matrix, there was only one box that was pushable upward/downward and only one box that was pushable rightward/leftward. Participants had to specify either the row or the column in which the specific box would be located after a push in the specified direction. After each matrix, the next cue appeared after a blank 
screen for $150 \mathrm{~ms}$. The sequence of priming and focus trials within a subblock was randomized.

Over the course of three subblocks, each target appeared once in each of the three priming conditions and additionally in the priming practice block. Within a subblock, each target was presented in one of the three priming conditions.

\section{Results}

Priming effects

Mean RTs were derived from correct responses to word targets. The mean error rate was $18.1 \%$. We discarded outlying RTs that were 1.5 interquartile ranges above the third quartile with respect to the individual RT distribution for each category type (Tukey, 1977); RTs that were above $1,500 \mathrm{~ms}$ or below $200 \mathrm{~ms}$ were discarded, irrespective of the individual outlier criterion (2.6\% of all trials). Mean RTs and mean error rates for word targets are depicted in Table 1; mean priming differences for word targets as a function of category type and focus task are presented in Fig. 2.

Mean priming effects (i.e., mean unrelated minus related RT differences) for RTs of word targets were subjected to a 2 (focus task: perception vs. action) $\times 2$ (category type: natural vs. artifactual) mixed ANOVA. ${ }^{2}$ The constant term was significant, $F(1,43)=5.38, M S E=735, p<.05$, indicating faster responses on average to related than to unrelated targets (i.e., a significant semantic priming effect). The main effects of category type and focus task were not significant (both $F_{\mathrm{s}}<1, p \mathrm{~s}>.63$ ). Importantly, there was a significant interaction between focus task and category type (see Fig. 2), $F(1,43)=6.81, M S E=534$, $p=.01$, showing that the focus task modulated the priming effects for natural and artifactual categories. To further clarify this interaction, we analyzed the priming effects for categories matching the current focus (i.e., natural/artifactual categories given a perception/action focus) and the priming effects for categories not matching the current focus (i.e., natural/artifactual categories given an action/ perception focus). Under a perception focus, the semantic priming effect for natural categories was significant, $t(22)=2.77, p=.01$, whereas the category priming effect for artifactual categories was not, $t(22)=0.34, p=.74$. Under an action focus, essentially the reverse pattern of results emerged: Artifactual categories elicited a significant

\footnotetext{
${ }^{2}$ In order to exclude interpretation of the effects as a result of repeating the priming material (i.e., the words and nonwords used as targets), we also analyzed the RTs from Subblock 1 of both the natural and artifactual blocks. These results were highly comparable to the results using the RT data from all subblocks. Therefore, an interpretations in terms of repetition effects can safely be excluded.
}

priming effect, $t(21)=2.11, p<.05$, whereas natural categories did not, $t(21)=0.19, p=.85$.

The same analyses on mean error rates revealed no significant effects (all $p \mathrm{~s}>.13$ ).

Focus tasks

Error rates and RT data (see Table 1) revealed that the focus tasks were of comparable difficulty. In particular, the focus tasks did not significantly differ in RTs or error rates (both $p$ s > .49).

\section{Discussion}

We analyzed whether a mind set can modulate semantic priming. Focus trials (inducing a mind set focusing on perceptual features or action features) were interspersed in a semantic priming experiment using natural and artifactual categories. Semantic priming effects emerged for natural categories only with a perception focus and for artifactual categories only with an action focus. The results support the ideas, first, that top-down factors influence automatic processes (see, e.g., Kiefer, 2007; Kiefer \& Martens, 2010) and, second, that conceptual representations are flexibly recruited (Hoenig et al. 2008). Not every aspect or feature of a concept is activated every time the concept is retrieved. Instead, the mind set can modulate which aspects are integrated into the current representation. Interestingly, the adaptation of concept representations occurred in a semantic priming paradigm, which was hitherto thought to reflect static relations between semantic concepts. Thus, we will first discuss below how our results fit into theoretical approaches on semantic priming. Our results are also of immediate relevance, however, for the debate on the representation of natural versus artifactual categories. Thus, we will also discuss the implications of our data for this topic.

Implications for theories on semantic priming

Older models of semantic priming (e.g., spreading activation; Anderson, 1983) offer no parsimonious way to integrate our results, but contemporary distributed network models do (e.g., Masson, 1995; Rogers \& McClelland, 2004). Distributed network models assume that every concept is represented by a set of processing units (i.e., distributed features) that are interconnected via weighted connections. Thus, a concept is represented by a specific stable pattern of activation across a set of processing units (e.g., Rogers \& McClelland, 2004). Facilitation due to semantic relatedness is explained in terms of the transition times between stable patterns. The transition from the 
Table 1 Mean reaction times (RTs, in milliseconds) and error percentages for word priming trials as a function of prime condition, category type, and focus condition, as well as mean RTs in the focus task as a function of focus condition (error percentages in parentheses)

\begin{tabular}{|c|c|c|c|c|c|c|c|c|}
\hline & \multicolumn{4}{|c|}{ Perception Focus } & \multicolumn{4}{|c|}{ Action Focus } \\
\hline & \multicolumn{2}{|c|}{ Related } & \multicolumn{2}{|c|}{ Unrelated } & \multicolumn{2}{|c|}{ Related } & \multicolumn{2}{|c|}{ Unrelated } \\
\hline & RT & $\% \mathrm{Er}$ & RT & $\% \mathrm{Er}$ & RT & $\% \mathrm{Er}$ & RT & $\% \mathrm{Er}$ \\
\hline Natural & 551 & 15.4 & 565 & 14.1 & 530 & 18.0 & 529 & 20.5 \\
\hline Artifactual & 537 & 16.8 & 538 & 18.8 & 509 & 22.2 & 521 & 22.2 \\
\hline Focus task & \multicolumn{4}{|c|}{ 4,181 (11.2) } & \multicolumn{4}{|c|}{$4,052(12.7)$} \\
\hline
\end{tabular}

prime's activation pattern to the activation pattern of the target can be carried out faster when the target is related to the prime, due to overlapping (and hence already activated) features. Having a perceptual focus might be conceptualized as parameters that facilitate the relaxation processes for parts of the vector representing perceptual features. Then, in the case of prime-target pairs that are dominantly related by shared perceptual/sensory properties - as is assumed for natural categories-larger priming effects are expected for participants with a perceptual focus. If prime-target pairs are mainly related by functional-feature overlap_as is assumed for artifactual categories - a focus on perceptual features will not cause facilitated target processing. In contrast, an action focus will lead to facilitated processes within the part of the network that represents the function of objects. Thus, the idea of dynamic recruitment of concepts due to different mind sets fits nicely into distributed network models of semantic priming. In addition, it should be noted that our data nicely fit recent observations using EEG measures of semantic priming, in which participants showed semantic priming only if they focused on semantic features, not if they focused on perceptual features (Kiefer \& Martens, 2010).

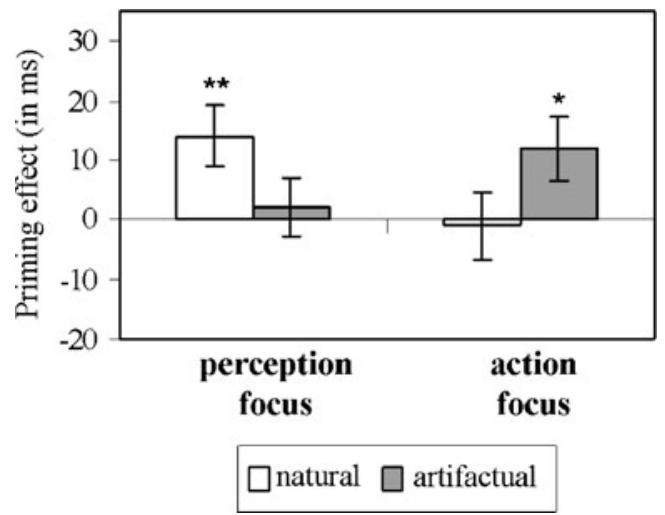

Fig. 2 Priming effects (in milliseconds) for natural and artifactual categories, as a function of perception or action focus. Error bars represent the standard errors of the means. ${ }^{*} p \leq .05 .{ }^{* *} p \leq .01$
Implications for the representation of natural versus artifactual categories

There is a long-standing debate on the distinction between the representations of natural and artifactual categories. It is clear from a vast array of studies using different experimental tasks, reporting both behavioral and neuroscientific data, that natural and artifactual objects can elicit different patterns of results (e.g., Martin \& Chao, 2001). A coarsegrained distinction in this body of work is between category-specific theories, which rely on separate categorical systems, and modality-specific theories, which rely mainly on the sensory-functional distinction. Categoryspecific theories assume that natural objects and artifactual objects are represented in different regions of the brain (e.g., Caramazza \& Shelton, 1998). Semantic knowledge is represented in these different regions in an amodal and abstract format; that is, knowledge about an object is represented separately from, for instance, action-related systems or perceptual systems. In contrast, sensoryfunctional theories support the idea that concepts are defined by sensory properties and motor properties (e.g., Hoenig et al., 2008; Kiefer, 2001, 2005; Sim \& Kiefer, 2005), both of which are acquired during experience. The processing of artifactual objects in semantic memory relies more on functional properties, whereas natural objects are represented there more on the basis of sensory properties. In addition, it is assumed that semantic knowledge is represented close to the sensory or motor cortex, depending on what region is active during acquisition (e.g., Humphreys \& Forde, 2001; Kiefer \& Spitzer, 2001). The retrieval of a specific concept also includes the reactivation of the proximate neurons that were active during acquisition.

With respect to our experiment, one could assume that, for example, an action (vs. perceptual) focus will activate the appropriate neurons in the motor (sensory) cortex, and hence that artifactual (natural) objects will profit from an action (perceptual) focus. Thus, our data clearly can be explained by sensory-functional theories, whereas category-specific theories - which assume fixed and differ- 
ent structures for natural and artifactual categories-cannot explain our results.

In summary, semantic priming effects for natural and artifactual categories depend on mind sets focusing on perceptual or functional features, respectively. Thus, our results support the idea of flexible concept representations and sensory-functional theories of category representation.

Author note The research reported in this article was supported by a grant from the Deutsche Forschungsgemeinschaft to D.W. and C.F. (WE 2284/5-2).

\section{References}

Anderson, J. R. (1983). A spreading activation theory of memory. Journal of Verbal Learning and Verbal Behavior, 22, 261-295.

Barsalou, L. W. (1982). Context-independent and context-dependent information in concepts. Memory \& Cognition, 10, 82-93.

Bermeitinger, C., Wentura, D., \& Frings, C. (2008). Nature and facts about natural and artifactual categories: Sex differences in the semantic priming paradigm. Brain and Language, 106, 153-163.

Caramazza, A., \& Shelton, J. R. (1998). Domain-specific knowledge systems in the brain: The animate-inanimate distinction. Journal of Cognitive Neuroscience, 10, 1-34.

Collins, A. M., \& Loftus, E. F. (1975). A spreading-activation theory of semantic processing. Psychological Review, 82, 407-428.

Gollwitzer, P. M., \& Kinney, R. F. (1989). Effects of deliberative and implemental mind-sets on illusion of control. Journal of Personality and Social Psychology, 56, 531-542.

Greenspan, S. L. (1986). Semantic flexibility and referential specificity of concrete nouns. Journal of Memory and Language, 25, 539-557

Hoenig, K., Sim, E. J., Bochev, V., Herrnberger, B., \& Kiefer, M. (2008). Conceptual flexibility in the human brain: Dynamic recruitment of semantic maps from visual, motor, and motionrelated areas. Journal of Cognitive Neuroscience, 20, 17991814.

Hutchison, K. A. (2003). Is semantic priming due to association strength or feature overlap? Psychonomic Bulletin \& Review, 10, 785-813.

Humphreys, G. W., \& Forde, E. M. E. (2001). Hierarchies, similarity, and interactivity in object recognition: "Category-specific" neuropsychological deficits. The Behavioral and Brain Sciences, $24,453-509$.

Kiefer, M. (2001). Perceptual and semantic sources of categoryspecific effects: Event-related potentials during picture and word categorization. Memory \& Cognition, 29, 100-116.

Kiefer, M. (2005). Repetition-priming modulates category-related effects on event-related potentials: Further evidence for multiple cortical semantic systems. Journal of Cognitive Neuroscience, 17, 199-211.
Kiefer, M. (2007). Top-down modulation of unconscious "automatic" processes: A gating framework. Advances in Cognitive Psychology, 3, 289-306.

Kiefer, M., \& Spitzer, M. (2001). The limits of a distributed account of conceptual knowledge. TRENDS in Cognitive Sciences, 5, 469471

Kiefer, M., \& Martens, U. (2010). Attentional sensitization of unconscious cognition: Task sets modulate subsequent masked semantic priming. Journal of Experimental Psychology: General, 139, 464-4889.

Martens, U., \& Kiefer, M. (2009). Specifying attentional top-down influences on subsequent unconscious semantic processing. Advances in Cognitive Psychology, 5, 56-68.

Martin, A., \& Chao, L. L. (2001). Semantic memory and the brain: Structure and processes. Current Opinion in Neurobiology, 11, 194-201.

Masson, M. E. J. (1995). A distributed memory model of semantic priming. Journal of Experimental Psychology. Learning, Memory, and Cognition, 21, 3-23.

McKoon, G., \& Ratcliff, R. (1995). Conceptual combinations and relational contexts in free association and in priming in lexical decision and naming. Psychonomic Bulletin \& Review, 2, 527533.

McNamara, T. P. (2005). Semantic priming: Perspectives from memory and word recognition. New York: Psychology Press.

McRae, K., \& Cree, G. S. (2002). Factors underlying categoryspecific semantic deficits. In E. M. E. Forde \& G. W. Humphreys (Eds.), Category specificity in mind and brain (pp. 211-249). Hove, U.K.: Psychology Press.

Neely, J. H. (1991). Semantic priming effects in visual word recognition: A selective review of current findings and theories. In D. Besner \& G. W. Humphreys (Eds.), Basic processes in reading: Visual word recognition (pp. 264-336). Hillsdale: Erlbaum.

Pecher, D., Zeelenberg, R., \& Raaijmakers, J. G. W. (1998). Does pizza prime coin? Perceptual priming in lexical decision and pronunciation. Journal of Memory and Language, 38, 401-418.

Rogers, T. T., \& McClelland, J. L. (2004). Semantic cognition: A parallel distributed processing approach. Cambridge: MIT Press.

Rothermund, K., Voss, A., \& Wentura, D. (2008). Counter-regulation in affective attentional biases: A basic mechanism that warrants flexibility in emotion and motivation. Emotion, 8, 34-46.

Sim, E. J., \& Kiefer, M. (2005). Category-related brain activity to natural categories is associated with the retrieval of visual features: Evidence from repetition effects during visual and functional judgments. Cognitive Brain Research, 24, 260-273.

Stevens, J. P. (1984). Outliers and influential data points in regression analysis. Psychological Bulletin, 95, 334-344.

Spruyt, A., De Houwer, J., \& Hermans, D. (2009). Modulation of automatic semantic priming by feature-specific attention allocation. Journal of Memory and Language, 61, 37-54.

Tukey, J. W. (1977). Exploratory data analysis. Reading: AddisonWesley.

Yeh, W., \& Barsalou, L. W. (2005). The situated nature of concepts. The American Journal of Psychology, 119, 349-384. 